\title{
Die Pseudoskorpion-Fauna (Arachnida: Pseudoscorpiones) eines Auwaldes bei Ingelheim am Rhein, unter besonderer Berücksichtigung der Auswirkungen des trocken-warmen Winters 2006/2007
}

\author{
Michael T. Marx, Oliver Weirich \& Gerhard Eisenbeis
}

\begin{abstract}
The pseudoscorpion-fauna (Arachnida: Pseudoscorpiones) of a floodplain close to Ingelheim/ Rhine, with special reference to the effects of the dry-warm winter 2006/2007. From the beginning of May 2005 to September 2007 the pseudoscorpion fauna in a hardwood floodplain forest of the Rhine valley near Ingelheim was investigated. Altogether 587 individuals representing two species from two families were captured using pitfall traps, trunk eclectors and by litter sieving. The warm, dry winter 2006/2007 exhibited a strong influence upon the activity of Neobisium carcinoides (Hermann, 1804). This winter event was followed by an extreme drought in April 2007, which affected the activity maximum of the corticolous species Chernes hahnii (C.L. Koch, 1839). In 2005 and 2006 the activity maximum of C. hahnii was observed in July, whereas in 2007 maturity was achieved earlier in May. Furthermore 31 individuals of N. carcinoides were captured in trunk eclectors. This climbing behaviour correlates with the presence of Lepidocyrtus lignorum (Collembola: Entomobryidae) on the trunks.
\end{abstract}

Keywords: activity, Collembola, drought, false scorpions, Germany, prey capture

Ökofaunistische Untersuchungen an Pseudoskorpionen sind in Mitteleuropa in den letzten Jahrzehnten sehr sporadisch durchgeführt worden. Erwähnt seien hier nur diejenigen von DROGLA (1990) (Deutschland; 349 Individuen), HELVERSEN (1966) (Deutschland; 554 Individuen) und MAHNERT \& HORAK (1994) (Österreich; 968 Individuen). BRAUN \& BECK (1986) werteten 3777 Pseudoskorpione aus, gesammelt im Rahmen bodenökologischer Studien im Schwarzwald. Im Jahr 2004 erschien dann eine ca. 23000 Individuen umfassende Darstellung zur Kenntnis der Pseudoskorpion-Fauna von Ostdeutschland (DROGLA \& LIPPOLD 2004). Diese Arbeit stellt die bisher umfangreichste Arbeit über Pseudoskorpione in Mitteleuropa dar, doch sollen hier die über Jahrzehnte durchgeführten Beobachtungen von RESSL (1983) nicht ungenannt bleiben. Es gibt nur wenige ökologische Arbeiten über die Pseudoskorpionfauna des Rhein-Main-Gebietes (HÖREGOTT 1963, HELVERSEN 1966). Die Ursachen sind nach DROGLA \& LIPPOLD (2004) in der geringen Anzahl an Bearbeitern zu sehen. Weitere Ursachen hierfür liegen sicherlich in der häufig unterschätzten ökologischen

\footnotetext{
Michael T. MARX, Gerhard EISENBEIS \& Oliver WEIRICH, Institut für Zoologie, Johannes Gutenberg-Universität Mainz / Abt.IV, 55099 Mainz; E-Mail:marxm1@students.uni-mainz.de,geisenbe@unimainz.de, oliweirich@gmx.de
}

eingereicht: 3.11.2007, akzeptiert: 31.1.2008
Bedeutung dieser Tiergruppe, sowie den niedrigen Fangabundanzen, welche in temperierten Zonen mit den standardökologischen Methoden erzielt werden können (GABBUTT 1970, YAMAMOTO et al. 2001). Als Grundlage für diese Arbeit standen Fänge aus Bodenfallen, Stammeklektoren und Streugesieben zur Verfügung, welche im Rahmen einer Untersuchung zur Arthropodenfauna eines Auwaldgebietes bei Ingelheim gewonnen wurden. Eine Hauptfragestellung dieser Untersuchung liegt in den Auswirkungen von extremer Trockenheit auf verschiedene Arthropodengruppen (Collembola, Araneae, Carabidae, Myriapoda sowie Opiliones) dieses Auwaldes. Dabei konnte auch eine relativ hohe Anzahl an Pseudoskorpionen gefangen werden, deren Auswertung einige Einblicke in die Ökologie dieser Tiergruppe in dem Auwaldhabitat erlaubt.

\section{Untersuchungsgebiet}

Das Untersuchungsgebiet ist eine Hartholzaue bei Ingelheim am Rhein. Der Auwald unterliegt regelmäßigen Frühjahrsüberflutungen unterschiedlicher Intensität. Jedoch blieb eine Vollüberflutung seit dem extremen Sommer 2003 aus. Trotz dieses Ausbleibens in den letzten Jahren kann der Auwald aufgrund seiner Nähe zum Rhein wohl noch als eher feuchtes Gebiet angesehen werden. Er liegt innerhalb des Mainzer Beckens und ist Teil des Naturschutzgebietes „Sandlache“, welches zugleich 
FFH-Gebiet ist. Das Klima ist als warm und trocken anzusehen (Jahresdurchschnitt 9,7 $\mathrm{C}, 500$ mm Niederschlag mit dem Hauptmaximum im Sommer). Eine genaue Beschreibung sowie eine schematische Karte des Gebietes (Fundort Nr. 5; TK25: 5914, 83 m ü. NN, 5000'N, 804'E) finden sich in MARX \& SCHÖNHOFER (2005). Als besonderes Klimaereignis ist der extrem trockene April $2007 \mathrm{zu}$ bewerten. Während dieser Periode fiel im Untersuchungsgebiet nur 0,2 $\mathrm{mm}$ Niederschlag und die Temperatur war um ca. $4^{\circ} \mathrm{C}$ wärmer, als das langjährige Mittel (Daten von AgrarMeteorologie, Rheinland-Pfalz).

\section{Material und Methoden}

Um die epigäische Bodenfauna des Auwaldes zu erfassen, wurden insgesamt 12 Bodenfallen nach BARBER (1931) eingesetzt. Die Fangtrichter in den Fallen hatten einen Durchmesser von jeweils $10 \mathrm{~cm}$. Gegen Regeneinfall wurden Plexiglasscheiben in einer Höhe von ca. $20 \mathrm{~cm}$ über den Fallen platziert. Weiterhin wurden jeweils im August der Jahre 2005-2007 Handaufsammlungen (Streugesiebe) durchgeführt, um Arten zu erfassen, welche in den Bodenfallen unterrepräsentiert waren. Dabei wurde innerhalb von 2 Stunden an festgelegten Stellen des Auwaldes die Bodenstreu gesiebt. Die hierbei gefangenen Individuen stellen nur Ergänzungen zu den Bodenfallen-Ergebnissen dar und fließen nicht in die phänologischen Betrachtungen der

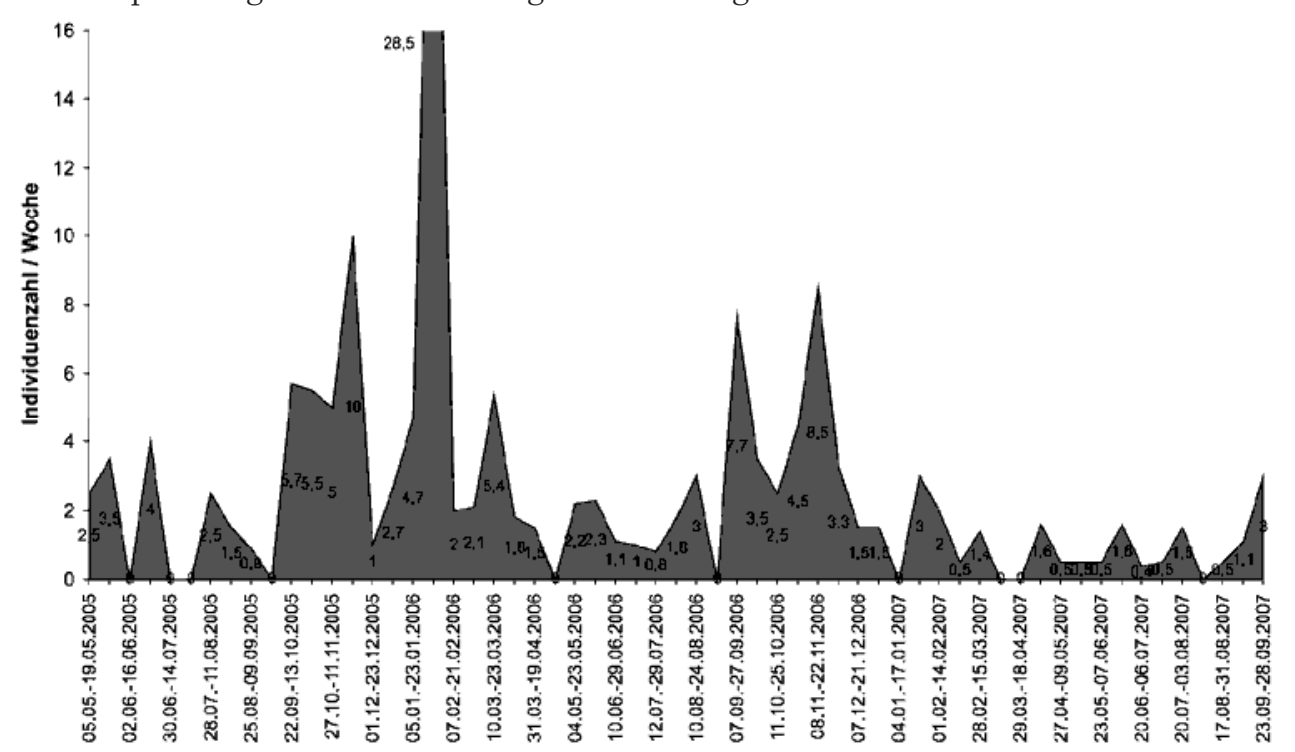

Abb.1: Durchschnittliche Individuenzahl pro Woche von N. carcinoides in den Bodenfallen über den gesamten Untersuchungszeitraum (5.2005-9.2007)

Fig.1: Average number of individuals per week of $N$. carcinoides in pitfall traps over the entire study period (5.2005-9.2007)
Arten ein. Um auch die atmobionte Fauna der Bäume zu erfassen, wurden zusätzlich 6 Stammeklektoren nach BEHRE (1989) verwendet, welche in einer Höhe von 1,70-2 m aufgehängt wurden. Dabei wurden unterschiedliche Baumarten mit einer entsprechend unterschiedlichen Rindenbeschaffenheit berücksichtigt. Die Stammeklektoren 1, 2 und 5 befanden sich an Stieleichen (Quercus robur), Stammeklektor 3 an einer Winterlinde (Tilia cordata), Stammeklektor 4 an einem Feldahorn (Acer campestre) und Stammeklektor 6 an einem Spitzahorn (Acer platanoides). Die Rinde der drei Stieleichen war tief gefurcht, die der Winterlinde relativ glatt, die des Feldahorns war im Bereich unterhalb des Stammeklektors abblätternd und die des Spitzahorns tief gefurcht aber nicht abblätternd. Bei allen verwendeten Fallen kam als Fangflüssigkeit gesättigte Salzlösung (ca. 30 \%) zum Einsatz. Um eine Vergleichbarkeit der unterschiedlichen Fangzeiträume zu gewährleisten, wurden die Individuenzahlen der Pseudoskorpione auf die durchschnittliche Individuenzahl pro Woche umgerechnet. Im Zeitraum vom 13.10.200527.10.2005 wurden die Stammeklektoren 1, 2, 3 und 6 sabotiert. Die Ergebnisse dieser Fangperiode beziehen sich somit nur auf die Stammeklektoren 4 und 5. Im Zeitraum vom 27.10.2005-11.11.2005 wurden alle Stammeklektoren zerstört und konnten infolge der Reparatur erst am 17.11.2005 wieder ausgebracht werden. 

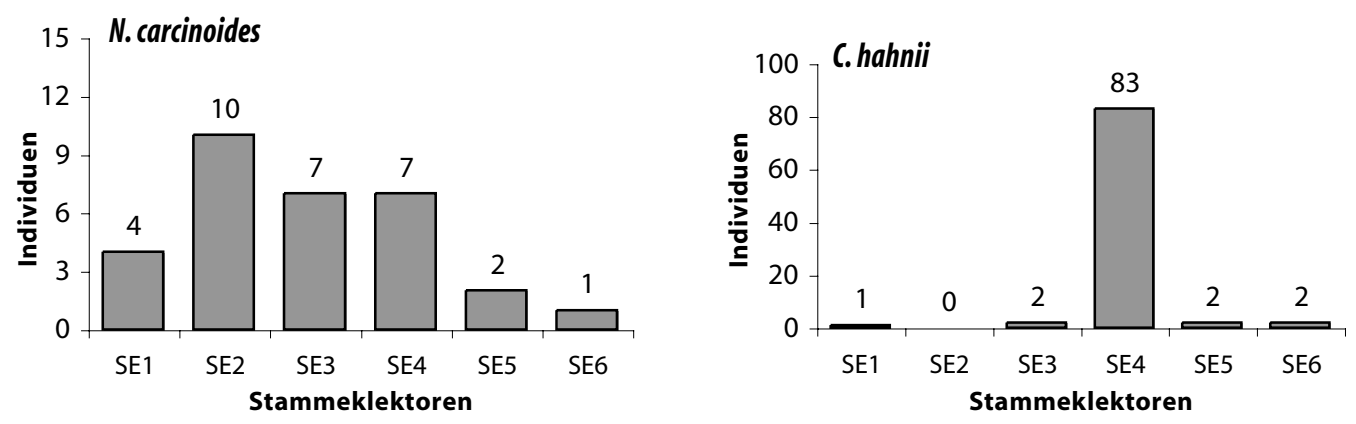

Abb.2: Gesamtindividuenzahlen von N. carcinoides und C. hahnii in den unterschiedlichen Stammeklektoren des gesamten Untersuchungszeitraumes (5.2005-9.2007)

Fig.2: Total number of individuals of $N$. carcinoides and C. hahnii of the different trunk eclectors over the entire study period (5.2005-9.2007)

Die Artbestimmung der Pseudoskorpione wurde mit BEIER (1963) durchgeführt. Bei der Bestimmung von Neobisium carcinoides $(=N$. muscorum) gab es im Schlüssel nach BEIER (1963) ein Problem. Hier darf das Tasthaar „ist"des festen Palpenfingers von „ib" nicht doppelt so weit entfernt sein, wie von der Fingerspitze. Es soll meist der Fingerspitze noch mehr genähert sein. Bei den meisten Individuen war das Tasthaar „ist" jedoch ziemlich genau doppelt so weit von „ib“entfernt, wie von der Fingerspitze. HELVERSEN (1966) weist allerdings auf genau dieses Problem hin. Nach seinen Erfahrungen gibt es neben einer häufigen mittelgroßen Form des $N$. carcinoides, auf welche die Beier'sche Beschreibung zutrifft, auch eine kräftige Form, bei der das Tasthaar „ist" meist von „ib“ doppelt so weit entfernt ist, wie von der Fingerspitze. Dafür, dass im Untersuchungsgebiet die kräftige Form auftritt, spricht auch, dass die Exemplare von $N$. carcinoides an feuchten Stellen im Allgemeinen größer sind als in trockenen Biotopen (RESSL \& BEIER 1958). MAHNERT (1988) betrachtete $N$. carcinoides als weit verbreitete polymorphe Art, die cytotaxonomischen Untersuchungen durch ŠŤÁHLAVSKÝ et al. (2003) sprechen jedoch für die Existenz von mehreren morphologisch kaum unterscheidbaren Arten.

Chernes habnii wurde zwar durch C.L. KOCH 1839 erstmals beschrieben, geriet dann aber in Vergessenheit und wurde lange Zeit mit Chernes cimicoides verwechselt und erst 1960 wieder entdeckt (BEIER 1960, 1963). Nach BEIER (1963) besitzen Arten der Gattung Chernes keine Augenflecken. Die in unserer Untersuchung gefundenen Chernetiden haben aber deutliche Augenflecke. Mahnert (pers. Mitt.) gibt ebenfalls an, dass $C$. hahnii entgegen Beiers Ausführungen undeutliche Augenflecke haben kann.

Die Artbestimmung der Collembolen erfolgte nach BRETFELD (1999), GISIN (1960) und HOPKIN (2007). Die Nomenklatur und Systematik der Arten richtet sich nach SCHULZ et al. (2005) und HOPKIN (2007).

\section{Ergebnisse}

Die Gesamtzahl der gefangenen Pseudoskorpione im Untersuchungszeitraum von Mai 2005 bis September 2007 betrug 587 Individuen aus zwei Arten und zwei Familien. Dabei wurden in Bodenfallen 323 Individuen von $N$. carcinoides und 2 Individuen von $C$. habnii gefangen.

Die durchschnittlichen Individuenzahlen von $N$. carcinoides pro Woche zeigten deutliche Aktivitätsmaxima von September-November 2005 und von Januar bis Februar 2006 (Abb. 1). Jedoch konnte das Aktivitätsmaximum in dem warmen und trockenen Winter des Jahres 2007 nicht erreicht werden. In den Handaufsammlungen traten ausschließlich Exemplare von $N$. carcinoides auf. Im August 2005 waren es 53 Individuen, während im August 2006 insgesamt 60 Individuen erfasst werden konnten. Im August 2007 waren es dann nur 28 Exemplare. In den Stammeklektoren konnten insgesamt 90 Individuen von C. hahnii und 31 Individuen von $N$. carcinoides erfasst werden.

$N$. carcinoides trat relativ homogen an den unterschiedlichen Bäumen auf (Abb. 2). Eine Präferenz für eine bestimmte Baumart ist nicht festzustellen. Anders verhält es sich bei $C$. hahnii. Hier wurden die meisten Tiere an einem Feldahorn (Acer campestre) erfasst. 


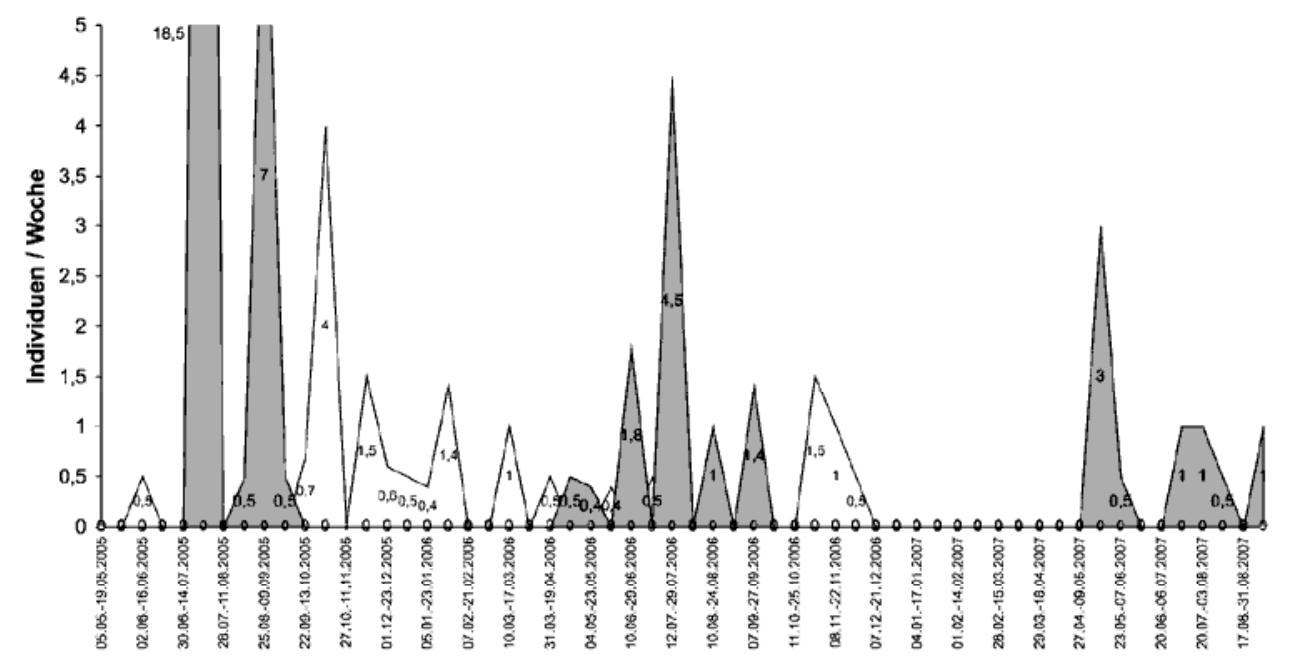

Abb.3: Durchschnittliche Individuenzahl pro Woche von N. carcinoides (grau) und C. hahnii (weiß) in den Stammeklektoren über den gesamten Untersuchungszeitraum (5.2005-9.2007)

Fig.3: Average number of individuals per week of $N$. carcinoides (grey) and C. hahnii (white) in trunk eclectors over the entire study period (5.2005-9.2007).

Im jahreszeitlichen Auftreten beider Arten ist eine deutliche Vikarianz festzustellen (Abb. 3). Sie konnten im Untersuchungszeitraum niemals gleichzeitig in den Stammeklektoren nachgewiesen werden. Diese negative Assoziation (COLE 1949) war signifikant (Chi ${ }^{2}$-Test, $\left.\mathrm{p} \leq 0,01\right)$. Weiterhin wurde der Einfluss des sehr warmen und trockenen Winters 2006/2007 auf das Auftreten von N. carcinoides deutlich. Während im Frühjahr 2006 noch kontinuierliche Fänge von $N$. carcinoides festzustellen waren, wurden ab Januar 2007 keine Exemplare dieser Art mehr in den Stammeklektoren nachgewiesen. Der extrem trockene April 2007 bewirkte bei C. habnii offenbar eine Verschiebung der Hauptaktivität in den Mai hinein. Des Weiteren konnte diese Art die hohen Individuenzahlen der beiden Vorjahre nicht mehr erreichen.

Es zeigt sich auf den ersten Blick kein deutlicher Zusammenhang zwischen dem Auftreten von $N$. carcinoides und der Dichte der Collembolen in den Stammeklektoren (Abb. 4, Abb. 6). Trennt man jedoch die typischen stamm- und baumbewohnenden Collembolen Entomobrya nivalis (Linnaeus, 1758), Orchesella cincta (Linnaeus, 1758) und Willowsia nigromaculata (Lubbock, 1873) (alle Entomobryidae), sowie den größeren bodenlebenden Kugelspringer Allacma fusca (Linnaeus, 1758) (Sminthuridae), der gelegentlich in hohen Dichten am Stamm anzutreffen ist, von der wesentlich kleineren epigäischen Art Lepidocyrtus lignorum
(Fabricius, 1775) (Entomobryidae) ab, so zeigt sich eine relativ deutliche Korrelation zwischen dem Stammauflauf von $N$. carcinoides und $L$. lignorum (Abb. 5, Abb. 7).

\section{Diskussion}

Das Auffinden von nur 2 Arten im Untersuchungsgebiet erscheint als gering, jedoch konnte auch HÖREGOTT (1963) insgesamt nur drei Arten (aus Bodenfallenfängen von 1954 bis 1957) in benachbarten Untersuchungsgebieten nachweisen. Dabei fand er insgesamt 84 Individuen, von denen 71 ebenfalls Neobisium carcinoides angehörten. Er konnte für diese Art im Untersuchungsgebiet eine Aktivitätsperiode von Dezember bis Januar und eine zweite im März aufzeigen. Diese zwei Perioden werden in dieser Untersuchung im Winter 2005/2006 bestätigt, jedoch zeigt sich hier eine dritte Aktivitätsperiode, welche schon im September beginnt und vor der zweiten Periode im Dezember wieder absinkt. Es spricht jedoch einiges für eine einzige plastische Winteraktivitätsperiode. Die unterschiedlichen Gipfel könnten witterungsbedingt entstehen. Deutlich ist das Ausbleiben der beiden Aktivitätsmaxima im Dezember 2006 und Januar 2007 sowie im März 2007 zu erkennen. Dieses Ausbleiben ist wahrscheinlich auf den zu warmen und trockenen Winter 2006/2007 zurückzuführen. Auch die Handfänge im Sommer bestätigen den Rückgang der Individuenzahl von 


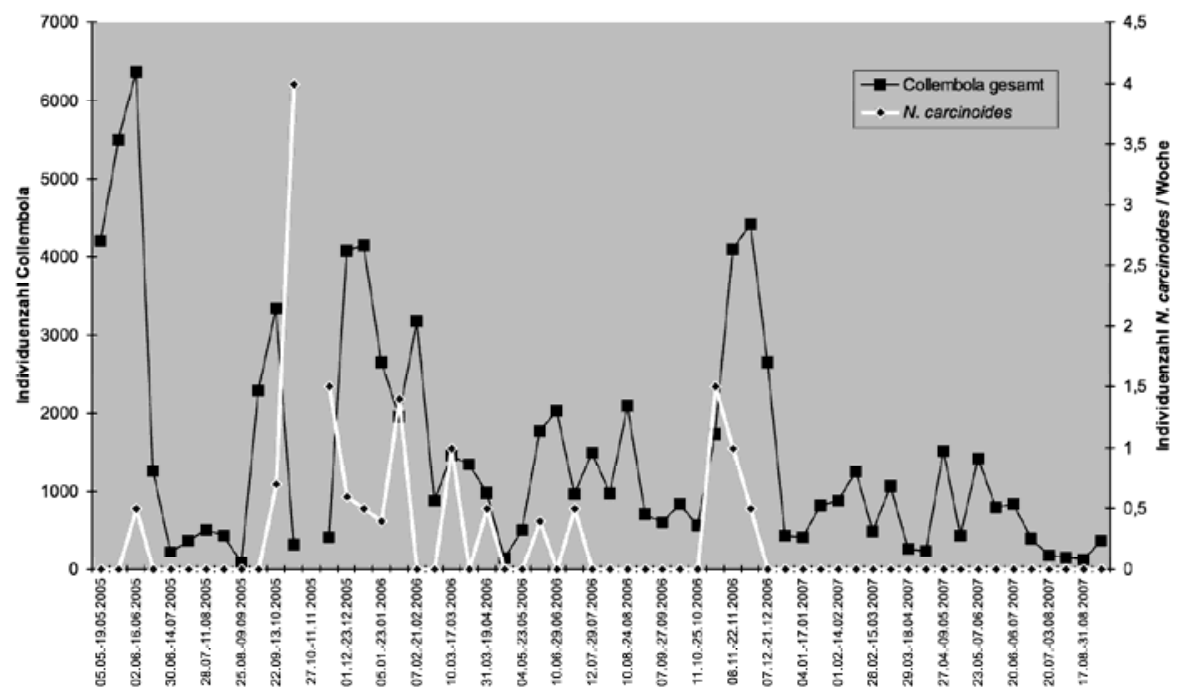

Abb.4:Vergleich der Individuenzahlen von N. carcinoides mit allen Collembolen

Fig.4: Comparison of the number of individuals of $N$. carcinoides and all Collembola

$N$. carcinoides nach diesem extremen Winter. Eingehende Angaben zur Phänologie von Neobisium carcinoides liegen aus dem Schwarzwald vor (BRAUN \& BECK 1986). Die Anpassungsfähigkeit von $N$. carcinoides an unterschiedliche Saisonalitäten und Temperaturen wurden auch schon von MEYER et al. (1985) in Nordtirol gezeigt. Dort gab es einen Wechsel des Lebenszyklus von zwei Generationen im Jahr in einem niedriger gelegenen Eichenmischwald zu einer Generation im Jahr in einem höher gelegenen Grünerlenbestand. Die Plastizität im Lebenszyklus von $N$. carcinoides (= N. muscorum) wurde ebenfalls in Großbritannien beschrieben (GABBUTT \& VACHON 1965, GABBUTT 1970, GODDARD 1976, 1979). Eine solche Temperaturverschiebung im Winter, gefolgt von einem extrem trockenen und warmen April 2007, scheint sich auf diese als hygrophil eingestufte Art (HEURTAULT \& VANNIER 1990) negativ auszuwirken. Karyologische Analysen von ŠŤÁHLAVsKÝ et al. (2003) führen zu einer möglichen weiteren Erklärung der verschiedenen Generationen. Die Variabilität der Chromosomen von $N$. carcinoides erlaubt die Folgerung, dass sich diese Art aus einem Komplex

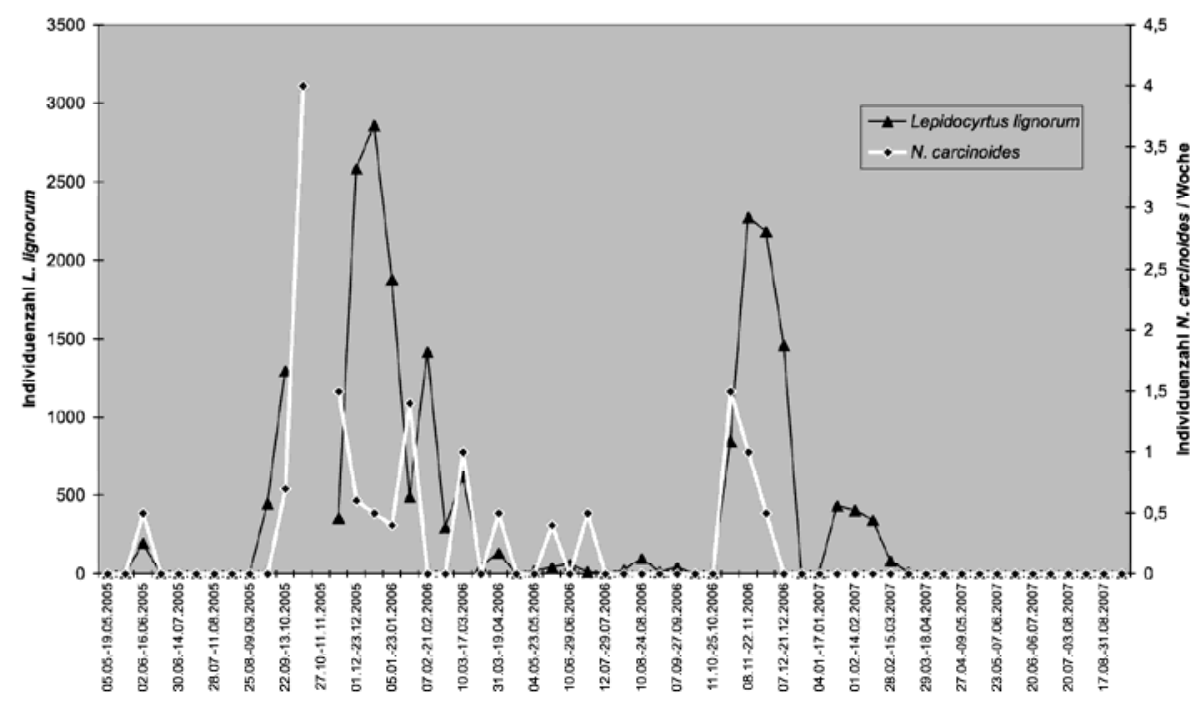

Abb.5: Vergleich der Individuenzahlen von N. carcinoides und L. lignorum.

Fig.5: Comparison of the number of individuals of $N$. carcinoides and L. lignorum. 


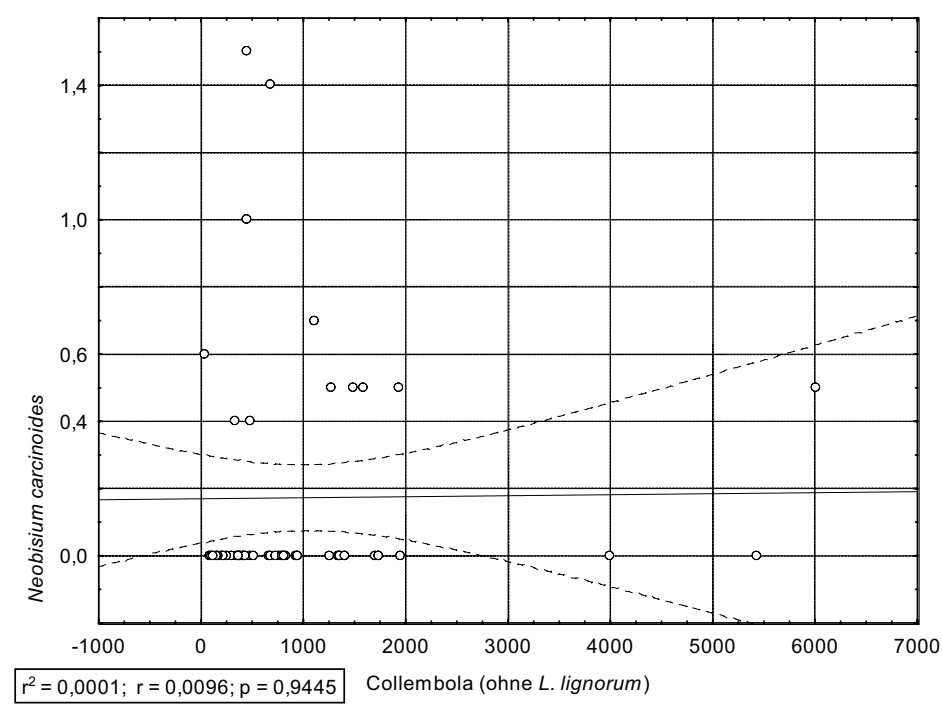

Abb.6: Korrelationsanalyse des Stammauflaufs von N. carcinoides und allen Collembolen (ohne Lepidocyrtus lignorum).

Fig.6: Correlation analysis of the vertical migration upon the trunk of Neobisium carcinoides and the total number of Collembola (except Lepidocyrtus lignorum).

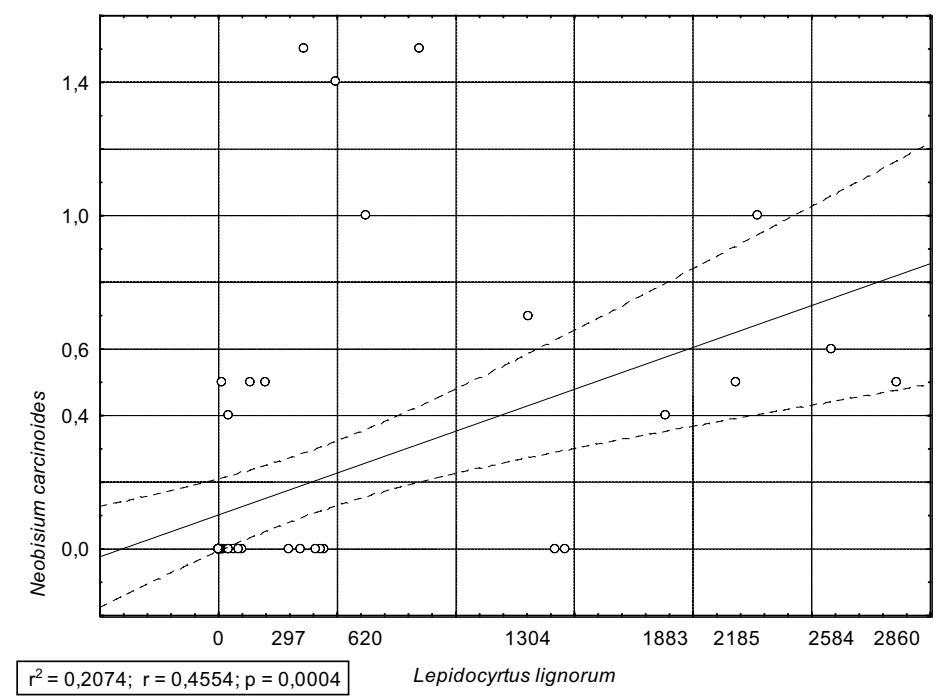

Abb.7: Korrelationsanalyse des Stammauflaufs von Neobisium carcinoides und Lepidocyrtus lignorum.

Fig.7: Correlation analysis of the vertical migration upon the trunk of Neobisium carcinoides and Lepidocyrtus lignorum.

von Arten zusammensetzt. Bisher konnten in Mitteleuropa drei kryptische Arten, welche jeweils konstante Chromosomenzahlen besitzen, ermittelt werden (ŠŤÁHLAVSKÝ et al. 2003).

Das häufige Auffinden von $N$. carcinoides in 1,5 bis
$2 \mathrm{~m}$ hoch angebrachten Stammeklektoren war so nicht zu erwarten. BRAUN \& BECK (1986) erwähnten zwar das Auftreten dieser Art im Fußbereich der Bäume, kein Fang erfolgte jedoch in den in etwa 1,8 m Höhe angebrachten BaumPhotoeklektoren. DRogLA \& LIPPOLD (2004) fanden bei über 10000 untersuchten Exemplaren insgesamt 5 Individuen in der Kraut- oder Strauchschicht und ein Individuum unter Baumrinde. Ein möglicher Erklärungsansatz dafür wäre die aktive Verfolgung der bevorzugten Beute (Collembolen) auf den Baumstamm. Genau wie bei den Collembolen ist bei $N$. carcinoides keine Präferenz für eine bestimmte Baumart oder Rindenbeschaffenheit zu erkennen. Die Neobisiiden gelten als spezialisierte Collembolenjäger (GILBERT 1951, WEYGOLDT 1969), jedoch können größere Collembolen nicht gepackt und mittels der Cheliceren zerquetscht werden (WEYGOLDT 1966). Vergleicht man das Vorkommen von $N$. carcinoides am Stamm mit den Gesamtindividuenzahlen der Collembolen, so ist auf den ersten Blick keine Übereinstimmung $\mathrm{zu}$ erkennen. Jedoch müssen die Collembolenfänge der Stammeklektoren differenziert betrachtet werden. Da $N$. carcinoides in diesem Erklärungsansatz der Beute folgt, müssen die typischen großen baumbewohnenden Collembolenarten wie Entomobrya nivalis, Willowsia nigromaculata und Orchesella cincta (alle Entomobryidae) ausgeklammert werden. Der Kugelspringer Allacma fusca (Sminthuridae) wäre zwar aufgrund seiner bevorzugten Lebensweise in der Streuschicht und dem periodischen Aufsteigen in die Stammregion in sehr großen Abundanzen ein idealer Kandidat für die Hypothese, jedoch dürfte er aufgrund seiner Größe von 3,5 mm nicht in das Beutespektrum von $N$. carcinoides fallen und muss 
somit ebenfalls ausgeklammert werden. Jedoch beschreibt BECK (1983) den 2,5-3 mm großen Kugelspringer Dicyrtoma ornata (Nicolet, 1842) als mögliche Beute von $N$. carcinoides. Diese Art wurde im Untersuchungsgebiet aber nicht am Stamm nachgewiesen. Eine weitere Collembolenart der Streuschicht, welche ebenfalls am Stamm empor klettert ist Lepidocyrtus lignorum (Entomobryidae). Diese Art ist ca. 1,6 mm groß und fällt somit eher in das Beutespektrum von $N$. carcinoides. Vergleicht man nun nur die Individuenzahlen dieser Collembolenart mit dem Auftreten von N. carcinoides am Stamm, so zeigt sich eine deutliche Korrelation. Damit könnten die hohen Individuenzahlen dieser Art am Stamm erklärt werden.

Während für $N$. carcinoides keine Präferenz für eine bestimmte Baumart oder Rindenbeschaffenheit festgestellt werden konnte, verhält sich Chernes habnii anders. Fast alle Individuen dieser Art fanden sich an einem Feldahorn mit abblätternder Rinde. DROGLA \& LIPPOLD (2004) fanden ebenfalls die meisten Individuen dieser Art an Platane und Ahorn. Anscheinend bevorzugt die Art die abblätternde Rinde dieser Baumarten, da sich durch diese Spalten mehr Lebensraum und Versteckmöglichkeiten bieten (vgl. RESSL 1983). C. hahnii bevorzugt nach HELVERSEN (1966) warme Standorte in offenem Gelände. BEIER (1960) zeigte weiterhin die Präferenz von C. hahnii für Bäume an Straßen und Flüssen, während die nahe verwandte Art C. cimicoides (Fabricius, 1793) geschlossene Wälder bevorzugt. WEYGOLDT (1969) berichtet von einer deutlichen Vikarianz beider Arten in Wäldern bei Berlin. Eine Vikarianz von C. bahnii und $N$. carcinoides am Stamm konnte in vorliegender Untersuchung zwar nachgewiesen werden; jedoch liegen die Aktivitätsmaxima beider Arten deutlich auseinander. Auch sind die bevorzugten Lebensräume sowie die Trockenresistenz beider Arten sehr verschieden, womit die Vikarianz auch auf andere Faktoren zurückzuführen sein könnte. Die Aktivitätsmaxima von C. hahnii liegen im Untersuchungsgebiet im Juli und September. Dies deckt sich mit den Angaben von HELVERSEN (1966), der die Fortpflanzungsperiode im Sommer nicht an bestimmte Monate gebunden sieht. Auffällig ist jedoch das vorverlegte Aktivitätsmaximum im Mai 2007 nach dem extrem trockenen und warmen April. Die Individuenzahlen lagen im Jahr 2007 auch beträchtlich unter denen der beiden Vorjahre.
Die extremen Witterungsverhältnisse scheinen sogar bei einer als xerophil eingestuften Art wie $C$. habnii (HEURTAULT \& VANNIER 1990) deutliche Auswirkungen zu zeigen. Weitere Untersuchungen über die Auswirkungen von extremen Trockenheiten auf diese Tiergruppe sind speziell im Hinblick auf die sich verändernden Klimabedingungen notwendig.

\section{Dank}

Das Projekt wurde finanziell unterstützt von der Feldbausch Stiftung und dem Kompetenzzentrum „Flut und Hitze" der Johannes Gutenberg-Universität Mainz. Großer Dank gilt auch den zwei Gutachtern sowie T. Blick sowie Dr. O.-D. Finch für die hilfreichen Kommentare und Verbesserungsvorschläge.

\section{Literatur}

BARBER H.S. (1931): Traps for cave-inhabiting insects. - J. Elisha Mitchell Sci. Soc. 46: 259-266

BECK L. (1983): Zur Bodenbiologie des Laubwaldes. - Verh. Dt. Zool. Ges. 1983: 37-54

BEHRE G. (1989): Freilandökologische Methoden zur Erfassung der Entomofauna (Weiter- und Neuentwicklung von Geräten). - Jb. Naturwiss. Ver. Wuppertal 42: 238-242

BeIER M. (1960): Chernes cimicoides (F.) und Chernes hahni (C.L. Koch), zwei gut unterschiedene Arten. - Z. Arbeitsgem. Österr. Entomol. 12: 100-102

BeIER M. (1963): Ordnung Pseudoscorpionidea (Afterskorpione). Bestimmungsbücher zur Bodenfauna Europas, Vol. 1. Akademie-Verlag, Berlin. 313 S.

BRAUn M. \& L. BECK (1986): Zur Biologie eines Buchenwaldbodens 9. Die Pseudoskorpione. - Carolinea 44: 139-148

Bretfeld G. (1999): Synopses on Palaearctic Collembola: Symphypleona. - Abh. Ber. Naturkundemus. Görlitz 71: 1-318

COLE L.C. (1949): The measurement of interspecific association. - Ecology 30: 411-424

Drogla R. (1990): Die Pseudoskorpione des Museums der Natur Gotha (Arachnida, Pseudoscorpiones). - Abh. Ber. Mus. Nat. Gotha 16: 97-102

DROGLA R. \& K. LiPPOLD (2004): Zur Kenntnis der Pseudoskorpion-Fauna von Ostdeutschland. - Arachnol. Mitt. 27/28: 1-54

GABbUTT P.D. (1970): Sampling problems and the validity of life history analyses of pseudoscorpions. - J. Nat. Hist. 4: 1-15

GABBUTT P.D. \& M. VACHON (1965): The external morphology and life history of the pseudoscorpion Neobisium muscorum. - Proc. Zool. Soc. London 145: 335-358 
GILBERT O. (1951): Observations on the feeding of some British false-scorpions. - Proc. Zool. Soc. London 121: 547-555

Gisin H. (1960): Collembolenfauna Europas. Mus. Hist. Nat., Genève. 312 S.

GODDARD S.J. (1976): Population dynamics, distribution patterns and life cycles of Neobisium muscorum and $C h-$ thonius orthodactylus (Pseudoscorpiones: Arachnida). - J. Zool. London 178: 295-304

GODDARD S.J. (1979): The population metabolism and life history tactics of Neobisium muscorum (Leach) (Arachnida: Pseudoscorpiones). - Oecologia 42: 91-105

Helversen O. VON (1966): Pseudoskorpione aus dem Rhein-Main-Gebiet. - Senck. Biol. 47: 131-150

Heurtault J. \& G. Vannier (1990): Modes de transpiration chez les Pseudoscorpions hygrophiles et xérophiles. - Bull. Soc. Eur. Arachnol. 1: 141-160

HÖREGOTT H. (1963): Zur Ökologie und Phänologie einiger Chelonethi und Opiliones (Arach.) des Gonsenheimer Waldes und Sandes bei Mainz. - Senck. Biol. 44: 545-551

HoPKIN S.P. (2007): A key to the Collembola (springtails) of Britain and Ireland. Field Studies Council, Totnes/Devon. $258 \mathrm{~S}$.

MAHNERT V. (1988): Neobisium carcinoides (Hermann, 1804) (Pseudoscorpionida, Neobisiidae) - une espèce polymorphe? - Bull. Soc. Sci. Bretagne 59, hors sér. 1: $161-174$

MAHNERT V. \& P. HORAK (1994): Distribution and ecology of pseudoscorpions (Arachnida: Pseudoscorpiones) in relict-forests in Styria (Austria). - Boll. Acad. Gioenia Sci.nat. 26: 245-252

MARX M.T. \&A. SCHÖNHOFER (2005): Abundanz und Vikarianz epigäischer Weberknechtarten (Arachnida: Opiliones) in einem Auwaldgebiet des Mainzer Beckens. - Arachnol. Mitt. 30: 13-19
MeYer E., H. WÄGER \& K. Thaler (1985): Struktur und jahreszeitliche Dynamik von Neobisium-Populationen in zwei Höhenstufen in Nordtirol (Österreich). - Rev. Écol. Biol. Sol 22: 221-232

RESSL F. (1983): Die Pseudoskorpione Niederösterreichs mit besonderer Berücksichtigung des Bezirkes Scheibbs, S. 175-202. In: RESSL F. (Hrsg.): Die Naturkunde des Bezirkes Scheibbs. Die Tierwelt des Bezirkes Scheibbs, Bd. 2. Radinger, Scheibbs. 584 S.

REsSL F. \& M. BEIER (1958): Zur Ökologie, Biologie und Phänologie der heimischen Pseudoskorpione. - Zool. Jb. Syst. 86: 1-26

SCHUlZ H.J., G. BRETFELD \& B. Zimdars (2005): Nomina Collembola Germanica. -Internet: http: //www.collembola.org/publicat/collgerm.htm (20.12.2007)

ŠŤÁHLAVSKÝ F., P. TƯMOVÁ \& J. KRÁL (2003): Karyotype analysis in Central European pseudoscorpions of the genus Neobisium (Pseudoscorpiones: Neobisiidae). S. 80. In: KIPYATKOV V.E. \& D.V. LOGUNOV (eds.): 21th European Colloquium of Arachnology. Program, abstracts, list of participants. St. Petersburg State Univ., Dept. Entomol. 104 S.

WEYGOLDT P. (1966): Moos- und Bücherskorpione. A. Ziemsen, Wittenberg Lutherstadt. $84 \mathrm{~S}$.

WEYGOLDT P. (1969): The biology of pseudoscorpions. Harvard Univ. Pr., Cambridge Massachusetts. $145 \mathrm{~S}$.

YAMAMOTO T., N. NOBUKAZU \& T. YOSHIFUMI (2001): Ecological study of pseudoscorpion fauna in the soil organic layer in managed and abandoned secondary forests. - Ecol. Res. 16: 593-601 